\title{
Building Brains for Robots: A Psychodynamic Approach
}

\author{
Andrzej Buller \\ Advanced Telecommunications Research Institute International \\ Network Informatics Laboratories, \\ 2-2-2 Hikaridai, Keihanna Science City, 619-0288 Kyoto, Japan \\ buller@atr.jp
}

\begin{abstract}
I argue that the disregard of the psychodynamic perspective by the Artificial Intelligence (AI) community is a kind of prejudice, and that AI/Robotics could benefit by dealing with some Freudian concepts. This point is supported by the results of some experiments with robots. An approach to AI/Robotics research called Machine Psychodynamics emerges from the discussion. The approach is compared with some other approaches in reference to mission, motivation, economy of behavior, world model, imitation, conflict resolution, role of perception, and human-robot communication.
\end{abstract}

\section{Introduction}

By an irony of fate the seemingly most insightful view of human mind, i.e., that proposed by Sigmund Freud and known as psychodynamic perspective, has never seen a serious debate in the Artificial Intelligence (AI) community. Indeed, the label "unscientific" could frighten away even the most open-minded seeker of strong AI. I argue that the disregard of the psychodynamic view is a kind of unfortunate prejudice, and, moreover, AI could benefit a lot by dealing with some Freudian concepts. This point is supported by some experimental results I briefly present. An approach to AI/Robotics research I call Machine Psychodynamics emerges from the discussion.

The psychodynamic perspective on the human mind (quite well represented in syllabi offered to students of social sciences) proposes that people's actions reflect the way thoughts, feelings, and wishes are associated in their minds; that many of these processes are unconscious; and that mental processes can conflict with one another, leading to compromises among competing motives [26, p. 15]. The key concepts in psychodynamics are tensions and defense mechanisms. Freud wrote: "The raising of these tensions is in general felt as unpleasure and their lowering as pleasure. It is probable, however, that what is felt as pleasure or unpleasure is not the absolute height of this tension but something in the rhythm of the changes of them." $[16, p$. 15]. Defense mechanisms are to keep an individual mentally balanced when a given tension cannot be reduced using the available repertoire of behaviors [5, pp. 184-5].

Psychodynamics is not to be confused with psychoanalysis. The latter is a theory and therapeutic method based on four assumptions: (i) the fundamental role of unconscious processes, (ii) the existence of conflicting mental forces and defense mechanisms, (iii) the existence of the Oedipus complex, and (iv) the key role of sexual drive and aggressive drive in the development of personality. It has been proposed that 
psychodynamics solely accepts the first two of the assumptions, whereas it does not consider the last two. It must also be said that in psychology there is still no universally accepted definition of the psychodynamic approach. For example, besides the obvious assumption about the influence of unconscious processes, Matt Jarvis [17, pp. 2-3] also proposes that the psychodynamic approach should assume the primacy of affect, the continuity between childhood and adult experience, the significance of early relationships, and the significance of subjective experience.

Perhaps psychodynamic concepts could not to date significantly influence the evolution of AI because of conclusions broadcasted by passionate critics of Freud and his work. Yet psychodynamic propositions "do not rise and fall with textual analysis of Freud's arguments. They rise and fall from evidence" [25]. Though Hans Eysenck and Glenn Wilson [15] provided devastating arguments against early attempts to verify Freudian theory empirically [19], several new results, obtained through proper scientific procedure, support basic Freudian concepts. Among several examples, Joel Weinberger and Drew Westen [25] quote literature from the last decade of the $20^{\text {th }}$ century dealing with object relations, in which investigators have coded hours of psychotherapeutic sessions and several projective responses, generally with reliabilities well above $r=.80$, and which have shown predictive validity with respect to a whole host of measures ranging from diagnosis to interpersonal behavior. They also quote longitudinal data showing that adult motivation positively correlates, as Freud hypothesized, with childhood training and parental permissiveness.

The myth about the "unscientific" character of the psychodynamics approach now faces a fast track to oblivion. After reviewing the recent findings in neurobiology vs. Freud's ideas, Eric Kandel, 2000 Nobel laureate, has concluded that psychoanalysis is "still the most coherent and intellectually satisfying view of the mind" [24]. Stuart Kaufmann [18] predicts that one day "psychoanalysis will be regarded as a forefront arena of science." There is no longer a reason to shy away from implementing selected psychodynamic concepts in artifacts, especially in view of the fact that the psychodynamic perspective is a severely expurgated version of Freudian work, i.e., free of the most controversial statements about sexuality and aggression.

\section{Psychodynamic Agent}

Psychodynamic concepts in related literature are provided in a narrative way. In order to make them useful for building artificial agents, they should be interpreted in more technical terms. It is virtually impossible to formulate definitions that are at once satisfactory or ultimate. So, let the following descriptions be treated as initial ideas facilitating the analysis of psychodynamic machines.

Tension is a physical quantity associated with a physical or simulated tensionaccumulating device. In such a device tension accumulates or discharges as a reaction to certain input signals. A collection of appropriately interconnected tensionaccumulating devices may constitute a memory.

Thoughts are meaningful patterns formed by states of tension-accumulating devices constituting working memory, i.e., the part of memory that the agent can more or less ably manipulate. 
Feelings are subjective experiences an agent can have when it is conscious and when certain elements of its working memory get important bodily signals and/or certain kinds of thoughts appear. In the case of unconscious artificial agents a given feeling may be substituted by a sum of signals produced by specialized devices connected to related tension-accumulating devices.

Pleasure is one of the feelings. It appears during a discharge of certain tensions. Even an unconscious agent can use a signal substituting pleasure as a reinforcing reward in training selected mechanisms.

Wishes are meaningful thought-like patterns in working memory representing imaginary objects or situations which are or were believed by the agent to possibly cause pleasure.

Conflicts are situations such that two or more contradictory wishes appear in working memory, while the agent can undertake an action in favor to only one of them.

The notion of working memory was introduced by Alan Baddeley and Graham Hitch [2] as a replacement for the dated notion of short-term memory, so it does not belong to the Freud legacy. Nevertheless, working memory well supplements the psychodynamic view as a platform for both conscious and unconscious processes, including a fight between conflicting thoughts and wishes.

Let psychodynamic agent mean an entity that meets or that can potentially meet the following assumptions:

i. The agent's only "mission" is to strive for its own pleasure.

ii. Pleasure comes from the discharge of tensions and its record is used as an award reinforcing the agent's learning.

iii. The agent's brain and body contain several tension-accumulating devices of various transfer functions working in such a way that given input signals produces a unique effect on the dynamics of changes of the level of the related tension; he signals are various spatiotemporal patterns coming from the environment or from the agent's brain or body; some of the signals represent states of other tension-accumulating devices.

iv. There are tensions that can have an effect on the agent's actuators, transfer functions of tension-accumulating devices, or on the growth of the neurons that form new devices.

v. The agent's sensors, actuators and tension-accumulating devices are configured in such a way that some tensions are always increasing and, in order to have pleasure, the agent more or less efficiently performs an action aimed at acquiring or producing signals capable of discharging the tensions.

vi. When two or more tensions become high at the same time, each of them tries to suppress all others and cause an action that can discharge it first.

vii. Some tension-accumulating devices form a memory that stores acquired knowledge and enables processing of the knowledge toward a tension-dischargeoriented action.

viii. When no action intended to discharge a given tension succeeds, then certain defense mechanisms may change the layout of the tension levels (thus giving the agent the opportunity to have a substitute pleasure from the discharge of another kind of tension) or modify the inconvenient memories.

ix. During the agent's development its memory system becomes sufficiently complicated to plan multi-step actions. 
x. Through interactions with its caregiver, the agent acquires the ability to judge other agents and social situations according to the criteria accepted in a particular society and becomes capable of feeling pleasure when it is positively judged by others.

Let us imagine a robot successfully built according to the above assumptions. First, it can be noted that it makes no sense to ask what its application is. A psychodynamic robot is a creature living its own life. This doesn't mean that it must be selfish and useless. In its simple version it may be a pet demonstrating a host of life-like behaviors. A developed psychodynamic robot, if properly raised, may feel satisfaction from successfully accomplishing tasks given by its human master.

The behavior of the psychodynamic robot is not necessarily caused by perception. For example, a lack of meaningful percepts may result in an increase of the tension labeled "boredom". In order to discharge this tension the robot explores its surroundings, which may result in encountering an object of interest and then acquiring signals discharging this tension. An more advanced psychodynamic robot may deliberately expose itself to inconveniences and dangers simply to accumulate related tensions and receive the pleasure that comes from discharging them.

When the robot learns a given behavior, or when its brain circuitry grows, pleasure-related signals serve as reinforcing awards. Hence, an appearance of specialized memories is expected in the growing brain. A long-term memory stores patterns representing acquired knowledge, thoughts and wishes, whereas a working memory enables interactions among the patterns. Via the interactions the robot generates plans of multi-step actions and tests them in its imagination before executing them. The robot can gain pleasure from a purely mental action, that is, when it manages to produce thoughts capable of serving as tension-discharging patterns.

The working memory may also serve as a theater in which conflicting beliefs or behavioral patterns may fight for access to long-term memory or actuators, respectively. The fight may end with a compromise or with a victory for the most promising idea; however, the victorious idea may after some time lose to a rival idea and then after more time win again, and so on [12].

Based on perceived and deduced facts, some sections of the memory system may form an inaccurate yet still useful model of surrounding world including the robot itself. The model may serve as a canvas for the evolution of models of desired reality and ideal reality. The differences between these two models may also cause tensions to be discharged and conflicts to be resolved. If the desired reality is based on the perceived behavior of observed humans or other robots, pleasure may come from the successful imitation of such behaviors.

\section{Some Experimental Results}

To test basic psychodynamic solutions one does not need a walking humanoid robot. A simple two-motor mobile vehicle with camera and speaker can demonstrate how tensions and pleasures work. Robots of such kind, Neko, Miao, and Miao-V, were used for the first experiments with tension-driven behaviors.

Neko was physically constructed. It had two wheels propelled by two dedicated motors, a speaker, color camera, and two touch sensors. Neko's brain consisted of functional blocks simulated on a cluster of three PCs and an on-board module for 
collision avoidance connected to the touch sensors. The implemented tensions represented boredom, excitation, fear, and a three-dimensional anxiety. Boredom increased when Neko did not perceive any object of interest and discharged when it saw one. When the camera detected a green object, the level of excitation increased and remained high as long as the object remained in the visual field. The level of fear became high immediately as a reaction to the appearance of a red object, remained high as long as the object remained in the visual field, and solely dropped after the object's disappearance. Anxiety was represented by the states of three tension-accumulating devices: $A n x_{L}, A n x_{R}$, and $A n x_{B}$. Each of the three parts of anxiety increased spontaneously and independently from the other. A discharge of $\mathrm{Anx}_{\mathrm{L}}, \mathrm{Anx}_{\mathrm{R}}$, or $\mathrm{Anx}_{\mathrm{B}}$ took place any time Neko turned left, right or back, respectively.

An arbitrary hardwiring determined the hierarchy of Neko's tensions. The priority list was: fear, excitation, anxiety, and boredom. The tension that achieved maximum volume suppressed the outputs of all tension-accumulating devices related to tensions located at lower positions at the list. As for the elements of the vector of anxiety, the one that first achieved maximum volume suppressed the outputs of the tensionaccumulating devices related to the other two.

An unsuppressed signal from a tension-accumulating device related to a tension of its maximum volume activated a dedicated functional module. The module activated by fear caused the robot to turn back and escape until fear fell below a defined level. The module activated by excitation forced the robot to chase the exciting object. In the case of activation of the module connected to Anxiety ${ }_{L}$, Anxiety A $_{R}$, or Anxiety ${ }_{B}$, Neko looked left, right, or back, respectively. When the signal produced by the tension-accumulating device representing boredom was high and unsuppressed, it went through a controlled associator connected to three functional modules: a scream generator, a driver for looking around, a driver for going forward. The associator initially activated the scream generator connected to the speaker. When for a defined time the associator did not receive a tension discharge signal, it activated the driver for looking around. If a tension discharge signal still did not come, the associator activated the driver for going forward. If even then a tension discharge signal did not come, it again activated the scream generator, and so on.

Equipped as described above Neko learned by itself how to cope with boredom that grew when no object of interest was perceived. It could choose between producing a sound, looking around, and going forward. Indeed, going forward increased the chance of seeing an object of interest. The learning was reinforced by a tension discharge signal. Since tensions representing "irrational" anxiety were also accumulated, in the event of a lack of an object of interest, Neko behaved as an animal in a cage, i.e. it wandered back and forth and "nervously" looked around [11].

Miao is a simulated creature living in a simulated world. Its tension-accumulation devices and functional modules cover fear-, excitation-, anxiety-, and boredomrelated behaviors similar to those demonstrated by Neko, as well as hunger-related behavior and a fight between hunger and excitation. The hunger volume is a function of the state of the simulated battery. If tensions representing fear and excitation are negligible, hungry Miao activates a module that drives it toward the battery charger. However, the activation signal must pass through a conflict-resolution device called MemeStorm. Also the signal representing excitation must pass through MemeStorm and only then can it activate the module that makes it chase the object of excitation. Inside MemeStorm, hunger and excitation try to suppress each other. The device has 
such an intrinsic dynamics that in the case of a substantial difference between competing tensions, the stronger one quickly wins, i.e., suppresses its rival and becomes high. However, when the competing tensions are close to a balance, one of the tensions wins, but after a couple of seconds loses to the other tension, with a possibility of winning again in a short time [20].

A related report states: Miao punches the ball / it stopped punching and looks toward the battery charger / Miao turns back to the ball and punches it (though not too vigorously) / suddenly it resigns, turns, and slowly approaches the charger / It gets very close to the charger / Miao sadly looks back at the ball, then turns and starts recharging... Isn't it life-like? Of course, the word "sadly", if treated literally, would be by all means farfetched. But what the experiment intended to show was not a "true" sadness. The point is that the robot's gaze looked sad and looked so not because somebody intentionally programmed a masquerade sadness, but because the robot's brain allowed for a psychodynamic process resulting in such expression.

Miao-V, like Miao, is a simulated creature living in a simulated world, with the same set of sensors, actuators, and tension-accumulating devices. However, the tensions that in the case of Miao represented fear, hunger, and excitation, in Miao-V represent desire for a red object, yellow object, and green object, respectively and increase randomly. Furthermore, unlike its predecessor, Miao-V has a brain that develops in a literal sense. Each new pleasure-related sensorimotor experience adds new cells and connections to its neural network. The network provides control signals to the speaker and to each of the two motors. What is essential is that there is no readymade circuitry for approaching an object of interest. A "newborn" Miao-V, like a newborn human baby, has no idea of how to purposefully use its actuators, so in the face of increasing tensions it can only produce random sounds and moves.

In the development of the brain of Miao- $V$ the role of its caregiver is fundamental. Hearing sequences of sounds produced by the robot, the caregiver gives it items she supposes it wants to get at the moment. If by accident the given item causes a discharge of the dominant tension, the subsequently generated pleasure signal reinforces changes in the neural network, thus increasing the strength of association between the tension and the most recently produced vocal expression. In this way, Miao- $V$ gradually learns to differentiate vocal expressions as distinguishable sequences of sounds, each dedicated to a different object of desire. At the same, time the caregiver gradually learns to properly guess the robot's needs based on the sequences of sounds she hears. In other words, within the pair-Miao-V and its caregiver-a common, mutually understandable proto-language emerges.

When, in this stage of the robot's brain development, the caregiver fails to give it a desired item, Miao- $V$ has no choice but to try and get the item itself. The growing network provides the motors with senseless signals, but, when by accident (or owing to a discrete caregiver's help) the item is touched, the related pleasure signal reinforces the changes in the network that caused the recent sequence of motor-driving signals and the locational changes of the image of the item in the robot's visual field. This way Miao- $V$ learns how to use its motors and camera to approach objects of desire with increasing efficiency. Having learned to approach immobile items, the robot then learned to chase mobile objects. When, having learned the art of approaching and chasing, Miao- $V$ still faces difficulties in catching the object of desire, it "recalls" the possibility of asking its caregiver to bring the object to it, so it again produces the appropriate sound sequences [21]. 
Although the brains of Neko and Miao can be expanded to cover more psychodynamic functionalities, because of the necessity of manually attaching the new tension-accumulating devices and behavior-generating blocks the constructions cannot be called psychodynamic agents. The above conclusion does not apply to Miao-V whose brain develops driven by pleasures.

\section{A New Discipline?}

This final section compares the psychodynamic approach to building brains for robots, labeled here Machine Psychodynamics, with some other approaches that I suppose might represent "mainstream AI/Robotics" in reference to such issues as: mission, motivation, economy of behavior, world model, imitation, conflict resolution, role of perception, and human-robot communication.

Mission. It seems natural that the purpose of research in the field of AI/Robotics is to build better and better devices that would better and better serve humans. Hence, anyone who presents a new construction or idea is asked: What is the application of the thing you propose? For what kind of missions has your robot been designed? It is seen as obvious that a robot must without reluctance accomplish missions instructed by its owner within an assumed area of application. Even the purchaser of an artificial pet seemingly assumes its full obedience. Machine Psycho-dynamics challenges this view of the human-robot relationship. Psychodynamic robots are assumed to live their own life and strive for pleasure for themselves. As long as robots are dependent on the resources we possess, it will be easy to use the resources as pleasurable awards and make psychodynamic robots do what we want.

Motivations. It seems to be commonly accepted that there is a hierarchy of goals and there are scales of values that guide the agent's decision-making process toward a particular goal (cf. [22, p. 192]). Another assumption, followed for example in the construction of Kismet [3, pp. 108-9], is that there are defined drives (social, stimulation, and fatigue) and the system should always try to maintain them in a bounded range called a homeostatic regime. Machine Psychodynamics does not deny the usefulness of goals and homeostatic regimes, however, it proposes that the most essential motivator is a measurable pleasure signal that is generated when a given tension is being discharged.

Economy of behavior. It seems natural that a robot to be deployed on the surface of a distant planet is designed to accomplish a defined mission in a possibly safe and economical way. The same applies to entertaining robots or even contemporary sociable robots. Nobody wants to witness a fall by his beloved robot (especially when it cost a fortune) from the table. Even a constructor of an advanced sociable robot would not appreciate a situation where the robot irrationally refuses to cooperate with children invited to her lab. Machine Psychodynamics challenges this economy-oriented way of thinking and admits the possibility that a robot may deliberately expose itself to inconveniences and dangers-just to accumulate a lot of tensions and have a great pleasure from discharging them. Stanislaw Lem once wrote a story whose characters debated on the possible reasons why one of their robots stupidly started climbing a rock, which resulted in a fatal fall. Did it do it for pleasure? Did it do it for curiosity? In psychodynamic terms the answer is obvious. 
World model. There is still no dominating attitude about the idea of a world model in mainstream AI/Robotics. For example Alexander Meystel and James Albus [22, p. 11] still see a world model as an indispensable block in the general scheme of intelligent systems. This view has been challenged by subsumption architecture [6] that is believed to enable the development of any robot behavior without building a world model. Based on empirical evidence it is argued that the human view of the surrounding world is never accurate and never complete [8]. Rodney Brooks convincingly argues that a robot may use the world as its own model [7, p. 42]. But for a roboticist who still has not given up the dream about machine consciousness the hypothesis of Richard Dawkins [13, p. 59] may be quite tempting: "Perhaps consciousness arises when the brain's simulation of the world becomes so complete that it must include a model of itself'. In view of the debate and the need to cover such phenomena as generating intentions and analyzing them versus socially acceptable rules, as well as generating plans and testing them in imagination, Machine Psychodynamics recommends that mechanisms for facilitating the emergence of a world model in a growing memory system be sought. The model can never become accurate and complete, but it may become more and more useful.

Imitation. Maybe the most attractive and efficient method of teaching people certain motor skills is demonstration. The same may apply to teaching robots, provided they have developed mechanisms for imitation. Hence, machine imitation is a hot topic in AI/Robotics. Humanoid robots are seen as the most suitable platforms for developing imitation mechanisms [4]. Kerstin Dautenhahn and Chrystopher Nehaniv [14, pp. 223] provide the "Big Five" central issues for designing experiments on imitation with autonomous agents: whom to imitate, when to imitate, what to imitate, how to map observed and imitated behavior, and how to evaluate the success. From the psychodynamic point of view, the authors omitted the most important issue: for what purpose to imitate? In Machine Psychodynamics imitation is seen as one of the ways of discharging tensions and, as a result, having pleasure. It was proposed [9] that an agent could be equipped with an innate mechanism that would create an imaginary desired scene in which it itself possesses the same item as a currently perceived individual or is doing the same things as the individual. The difference between the perceived and desired reality may cause a tension that can be discharged when the perceived reality becomes similar to the desired reality, which would be a drive to imitate. In further development, the agent could achieve the ability to cease imitating if it violated socially accepted rules or endangered its more essential interests.

Conflict resolution. A conflict appears when two or more behavior-generating modules attempt to drive a robot's actuators. Ronald Arkin [1, pp. 111-9] presents two approaches to behavior coordination: (a) using competitive methods that decide about output by arbitration or action-selection and (b) using cooperative methods that find a superposition of forces or gradients based on the notion of field. In both cases, the point is to work out in a reasonable time an explicit decision of what the robot is to do. This works well in the case of robots with a defined area of application. Nevertheless, it can be noted that such "self-confident" decisiveness is not life-like.

One of the conflict-related psychological phenomena is the so-called mouse effect. Human subjects were asked to read a story about a controversial person and then to express their feelings using a computer mouse. Positive feelings were to be expressed by locating the cursor at the center of the screen, whereas the cursor located at the border of the screen would mean highly negative feelings. The records of cursor 
locations revealed a counterintuitive truth about social judgment. Even if no new data about the person of interest were heard, it occurred that after a period of keeping the cursor near the screen center a subject suddenly moved it to the screen's border [23, pp. 97-98]. Therefore, Machine Psychodynamics recommends developing mechanisms that enable conflicting ideas to fight, with the possibility that the victorious idea may after some time lose to another idea and after some time win again [10][20]. This is the way to a life-like machine ambivalence and hesitation that, as Andrzej Nowak and Robin Vallacher [23, pp. 101-2] suppose, play a non-negligible role in cognitive development.

Communication. One can often see demonstrations of so-called "communicating robots" that "speak" pre-recorded sentences. This obvious masquerade is one of the by-products of mainstream AI/Robotics. More ambitious constructions use speech generators to articulate sentences generated by programs manipulating strings of symbols toward the more or less efficient parsing of perceived questions and a knowledge-based synthesis of sensible answers. A different sort of research is aimed at developing mechanisms for a growth of the ability of meaningful communication through social interactions reinforced by simulated motivations. Machine Psychodynamics goes in a similar direction, however, it assumes that communication behaviors (including natural language) are to emerge from pleasure-oriented interactions between a robot and its caregiver.

\section{Acknowledgement}

This research was supported by the National Institute of Information and Communications Technology of Japan (NICT), Grant 13-05. The described experiments used the tools and models engineered by Juan Liu, Michal Joachimczak, Daniel Jelinski, Beata Grzyb, Adam Stefanski, and Tarun Tuli.

\section{References}

1. Arkin, R.: Behavior-Based Robotics. The MIT Press, Cambridge, Mass. London (1998).

2. Baddeley, A.D., Hitch, G.J.: Working Memory. In Bower G.A. (ed.): The Psychology of Learning and Motivation. Academic Press, New York (1974) 47-89

3. Breazeal, C. L.: Designing Sociable Robots. A Bradford Book/The MIT Press, Cambridge, Mass. London: (2002)

4. Breazeal C.L. \& Scassellati B.: Robots that imitate humans. Trends in Cognitive Sciences, 6 (11) (2002) 481- 487

5. Brody, N. \& Ehrlichman, H.: Personality Psychology: The Science of Individuality. Prentice Hall, Upper Saddle River, NJ (1988)

6. Brooks, R: A Robust Layered Control System For a Mobile Robot. In Brooks R.: Cambrian Intelligence: The Early History of the New AI. A Bradford Books/The MIT Press, Cambridge, Mass. London (1999) 3-26

7. Brooks, R.: Flesh and Machines: How Robots Will Change Us. Pantheon Books, New York (2002)

8. Brooks R.A., Breazeal (Ferrel) C., Irie R., Kemp C.C., Marianović M., Scassellati B., Williamson, M.M: Alternative Essences of Intelligence. AAAI-98 (1998) 961-968

9. Buller, A.: Psychodynamic robot. Proc. FIRA Robot World Congress, Seoul (2002) 26-30 
10. Buller, A.: Dynamic Fuzziness. In Ishizuka M., Satter A.: PRICAI 2002: Trends in Artificial Intelligence, Proc. of the $7^{\text {th }}$ Pacific Rim Int. Conf. on Artificial Intelligence, Tokyo, Lecture Notes in Artificial Intelligence, Vol. 2417. Springer-Verlag, Berlin (2002) 90-96

11. Buller, A.: From q-cell to artificial brain. Artificial Life and Robotics, Vol. 8 (1) (2004) 89-94

12. Buller A., Shimohara K.: On the dynamics of judgment: does the butterfly effect take place in human working memory? Artificial Life and Robotics, Vol. 5 (2) (2001) 88-92

13. Dawkins, R.: The Selfish Gene. Oxford University Press, Oxford (1976/1989)

14. Dautenhahn, K., Nehaniv Ch.: Imitation in Animals and Artifacts. A Bradford Books/The MIT Press. Cambridge, Mass., London (2002)

15. Eysenck, H., Wilson, G.: The Experimental Studies of Freudian Theories. Barnes \& Noble, New York (1974)

16. Freud, S.: An Outline of Psycho-Analysis. W.W. Norton, New York (1940/1989)

17. Jarvis, M.: Psychodynamic Psychology. Classical theory and contemporary research. London, Thomson (2004)

18. Kauffman, S.: The emergent ego. In Palombo S.R.: The Emergent Ego: Complexity and Coevolution in the Psychoanalytic Process. Int. Univ. Press, Madison (1999) xii

19. Kline, P.: Fact and Fancy in Psychoanalytic Theory. Methuen, London (1972)

20. Liu, J., Buller, A.: Tensions and conflicts: Toward a pleasure-seeking artifact. $5^{\text {th }}$ IFAC Symposium on Intelligent Autonomous Vehicles (IAV'2004). Lisboa, Portugal (2004)

21. Liu, J. \& Buller, A.: Emergent communication and motor behavior of a simulated robot. Unpublished research note (2004)

22. Meystel, A.M., Albus, J.S.: Intelligent Systems. Architecture, Design, and Control. John Wiley \& Sons, New York (2002)

23. Nowak, A., Vallacher, R.A. Dynamical Social Psychology. Guilford Press, New York (1998)

24. Solms, M.: Freud returns. Scientific American Vol. 290 (5) May, (2004) 82-8

25. Weinberger, J., Westen, D.: Science and Psychodynamics: From Arguments About Freud to Data. Psychological Inquiry 12 (3) (2001) 129-132

26. Westen, D.: Psychology. Mind, Brain, \& Culture. Second Edition. John Wiley \& Sons. New York (1999) 